\title{
Conical diffraction: observations and theory
}

\author{
By M. V. Berry ${ }^{1, *}$, M. R. Jeffremer ${ }^{1}$ and J. G. Lunney ${ }^{2}$ \\ ${ }^{1}$ H. H. Wills Physics Laboratory, Tyndall Avenue, Bristol BS8 1TL, UK \\ ${ }^{2}$ Physics Department, Trinity College Dublin, College Green, Dublin 2, Ireland
}

Conical refraction was produced by a transparent biaxial crystal of $\mathrm{KGd}\left(\mathrm{WO}_{4}\right)_{2}$ illuminated by a laser beam. The ring patterns at different distances from the crystal were magnified and projected onto a screen, giving rings whose diameter was $265 \mathrm{~mm}$. Comparison with theory revealed all predicted geometrical and diffraction features: close to the crystal, there are two bright rings of internal conical refraction, separated by the Poggendorff dark ring; secondary diffraction rings decorate the inner bright ring; as the distance from the crystal increases, the inner bright ring condenses onto an axial spot surrounded by diffraction rings. The scales of these features were measured and agreed well with paraxial theory; this involves a single dimensionless parameter $\rho_{0}$, defined as the radius of the rings emerging from the crystal divided by the width of the incident beam. The different features emerge clearly in the asymptotic limit $\rho_{0} \gg 1$; in these experiments, $\rho_{0}=60$.

Keywords: polarization; birefringence; crystal optics; conical refraction

\section{Introduction}

The phenomenon of conical refraction (Born \& Wolf 1959) was predicted as long ago as 1832 (Hamilton 1837; O'Hara 1982) and observed soon afterwards (Lloyd 1833). It played an important part in the development of optics, not only by speeding the acceptance of the transverse wave nature of light, but also, in retrospect, as one of the first optical singularities (Berry 2000). More generally, conical refraction was influential in theoretical physics, as Hamilton's first concrete application of his concept of phase space. Nevertheless, comparisons of theory with experiment have been scanty.

Even the definitive formulation of the theory (Belskii \& Khapalyuk 1978; Belsky \& Stepanov 1999) was relatively recent, and depended on the recognition of two principles: first, that details of the field emerging from the crystal can be understood only in terms of waves-hence our term 'conical diffraction'; and second, that the analysis can be greatly simplified by incorporating paraxiality (all deflection angles small). Exploration of the content of the theory continues (Berry 2004) with the derivation of analytical formulae for three features of conical diffraction: the two bright rings separated by the Poggendorff dark ring (previously explained in qualitative terms by Voigt (1905a)); the secondary inner rings (previously observed in numerical computations by Warnick \& Arnold

* Author for correspondence (tracie.anderson@bristol.ac.uk). 
(1997)); and the transition from internal to external conical refraction as the observation distance increases (previously noted in observations by Raman (1942) and Raman et al. (1941)).

There have been a few experimental confirmations of conical refraction (Lloyd 1833; Poggendorff 1839; Raman et al. 1941), including a spectacular demonstration (Perkalsris \& Mikhailichenko 1979; Mikhailichenko 2004) and at least two quantitative comparisons with theory (Schell \& Bloembergen 1978a; Fève et al. 1994) for the two bright rings. However, the difficulty of obtaining biaxial crystals of sufficient quality and size, together with the late development of theory, has delayed comprehensive identification of all the associated phenomena.

Our aim here is to exploit our recent acquisition of a transparent biaxial crystal of sufficiently high quality to provide this identification and make quantitative comparisons between theory and experiment. We begin (§2) by describing our observations and showing digital photographs of the conical diffraction patterns beyond the crystal, illustrating the different phenomena in a qualitative and non-technical manner. Then (§3), we give an outline of the theory, by presenting the formulae (Belsky \& Stepanov 1999; Berry 2004) in a simplified form, and without derivation, alongside a description of the phenomena they describe. We emphasize the fact that the field beyond the crystal depends on a single parameter $\rho_{0}$ : the radius of the cylinder emerging from the crystal divided by the width of the incident light beam. Section 4 contains analyses of the photographs, comparing the scales of different features of the patterns with the predictions of theory. Without the paraxial approximation, the theory would be much more complicated; paraxiality is analysed in appendix A, where it is shown that differences between geometrical features of the patterns calculated paraxially and those of the exact theory are indiscernible in our experiments and in all others so far reported.

\section{Observations}

At the heart of the experimental arrangement (figure 1) is a biaxial crystal of $\mathrm{KGd}\left(\mathrm{WO}_{4}\right)_{2}$, belonging to the monoclinic double tungstate family, manufactured by the company Vision Crystal Technology AG. The crystal has the shape of a matchstick $25 \mathrm{~mm}$ long, with its cross-section $3 \mathrm{~mm}^{2}$, and is cut so that an optic axis lies close to the long direction. Circularly polarized light from a $\mathrm{He}-\mathrm{Ne}$ laser (wavelength $632.8 \mathrm{~nm}$ ) was focused onto the crystal, the emerging light was projected, magnified, onto a distant screen, and was photographed with a Fuji F610 digital camera.

Unless the crystal is accurately oriented, the incident beam is split into the two beams characteristic of double refraction, visible as spots on the screen (figure 2a). As the crystal is rotated so that its optic axis is brought into alignment with the incident beam, the spots deform into lunes (figure $2 b$ ) and then merge (figure $2 c$ ) to form the two rings of internal conical refraction (figure $2 d$ ); these are the intersections with the observation plane of the two coaxial cylinders of bright light into which the incident beam has been transformed. This transition has been explored in detail (Lloyd 1837; Dreger 1999); we show it here for completeness. 


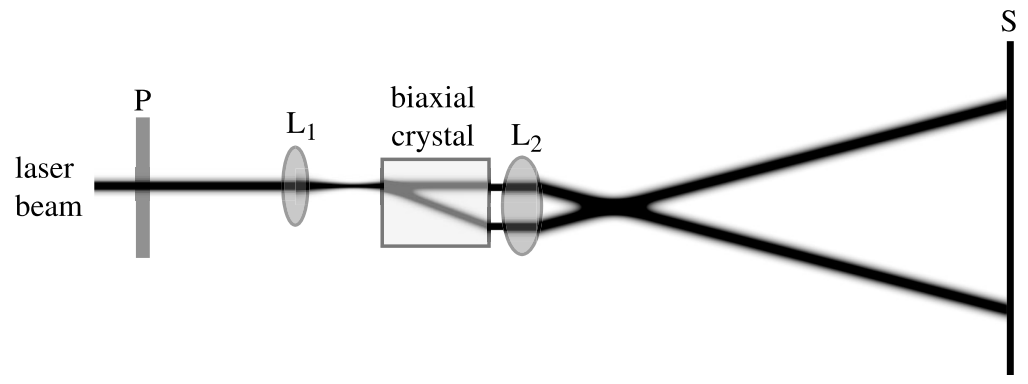

Figure 1. Arrangement for observing conical diffraction. A collimated laser beam passes through a circular polarizer $\mathrm{P}$, is focused by the lens $\mathrm{L}_{1}$ (focal length $70 \mathrm{~mm}$ ) and enters a biaxial crystal of $\mathrm{KGd}\left(\mathrm{WO}_{4}\right)_{2}$ of length $25 \mathrm{~mm}$. The emerging cylinder of light, magnified by the lens $\mathrm{L}_{2}$ (focal length $6.4 \mathrm{~mm}$ ), is observed on the screen $\mathrm{S}, 2 \mathrm{~m}$ from the crystal.
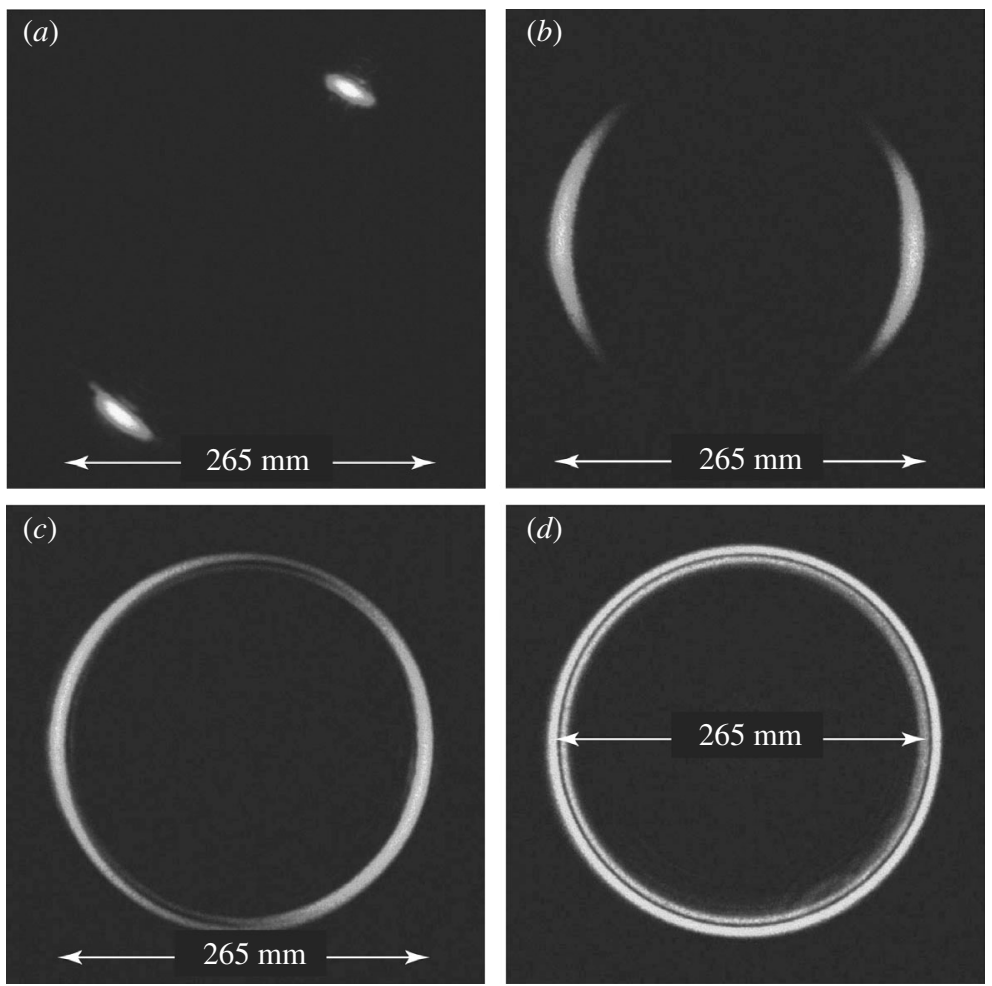

Figure 2. Transition from $(a)$ double refraction to $(d)$ conical refraction as the crystal is rotated until the incident beam is parallel to an optic axis.

With the incident beam aligned with the optic axis, the lens $\mathrm{L}_{2}$ was moved so as to project onto the screen observation planes at different distances from the crystal. Figure $3 a$ shows the narrow, sharp rings near the plane within the crystal where the incident beam waist is imaged. Receding from this plane (figure $3 b$ ), the rings get broader; the outer ring is fainter and the inner, brighter ring acquires structure in the form of fine inner fringes; these are the 'secondary rings' previously noted in numerical calculations by Warnick \& Arnold (1997). 

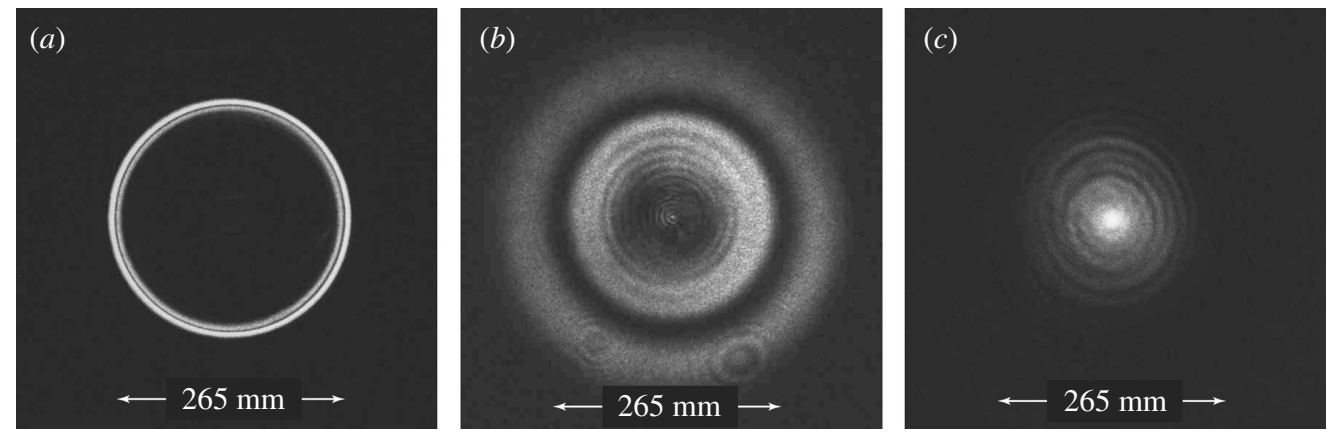

Figure 3. Conical diffraction patterns at increasing distances from the crystal. (a) Very close to the focal image plane inside the crystal; (b) $12 \mathrm{~mm}$ from the focal plane; $(c) 49 \mathrm{~mm}$ from the focal plane.

Receding further (figure 3c), the outer ring disappears, and the inner ring collapses into a bright central spot surrounded by fine circular fringes; this is related to (and near the crystal exit face corresponds to) the spot of external conical refraction noted in experiments by Raman et al. (1941).

In our experiments, we used circularly polarized incident light, for which the emerging light intensity has circular symmetry; it is the detailed radial structure of this pattern that concerns us here. We also made observations with linearly polarized light, and observed the resulting characteristic azimuthal modulation of the intensity; since this is well understood (Hamilton 1837), we do not report these observations. We note, however, that the polarization pattern has interesting implications for optical angular momentum (Berry et al. 2005).

\section{Recapitulation of theory (Belskii \& Khapalyuk 1978; Belsky \& Stepanov 1999; Berry 2004)}

In attempting to interpret the observations in the light of theory, we begin by considering a transparent biaxial non-chiral linear crystal, with three distinct principal refractive indices

$$
n_{1}<n_{2}<n_{3},
$$

and assuming that the differences $n_{2}-n_{1}$ and $n_{3}-n_{2}$ are small compared with $n_{2}$, so that all refraction and diffraction effects are paraxial (see appendix A). As predicted by Hamilton (1837), a collimated beam of light spreads within the crystal into a narrow hollow cone with a semiangle

$$
A=\frac{1}{n_{2}} \sqrt{\left(n_{2}-n_{1}\right)\left(n_{3}-n_{2}\right)}
$$

We consider the case where the incident beam, with vacuum wavenumber $k_{0}$, is circularly symmetrical and Gaussian, with the transverse profile of the electric field magnitude, in the beam waist (width $w$ ) given by

$$
E_{0}(r)=\exp \left(-\frac{r^{2}}{2 w^{2}}\right)
$$




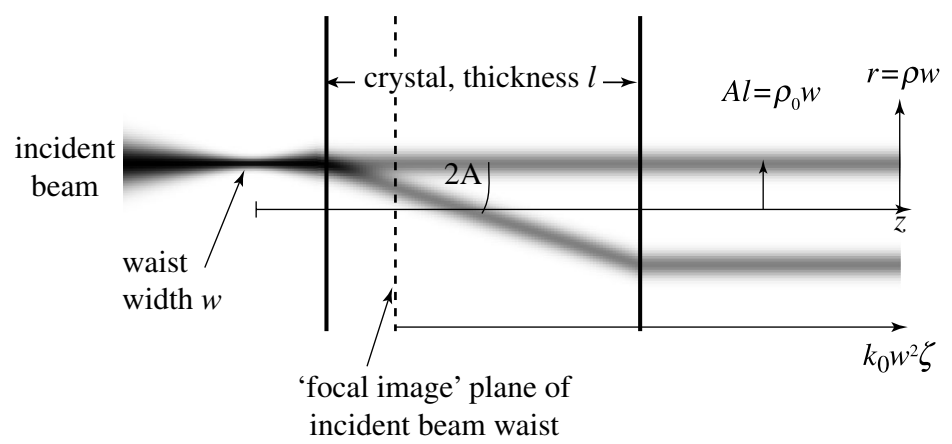

Figure 4. Geometry and coordinates for incident beam, cone inside the crystal and cylinder emerging from the crystal (schematic).

The spectrum of plane waves comprising this beam is also Gaussian, with the plane waves filling a cone of directions whose semiangle is $1 /\left(k_{0} w\right)$.

The profile of the beam that emerges after passing through thickness $l$ of the crystal (figure 4) depends on the following dimensionless variables:

$$
\rho_{0} \equiv \frac{A l}{w}, \quad \rho \equiv \frac{r}{w}, \quad \zeta \equiv \frac{l+(z-l) n_{2}}{n_{2} k_{0} w^{2}},
$$

where $r$ and $z$ are cylindrical polar coordinates, with $r$ measured from the axis of the emerging cylinder and $z$ measured from the waist of the incident beam.

We emphasize the significance of $\rho_{0}$. This single parameter, incorporating the influence of the five physical quantities $n_{1}, n_{2}, n_{3}, l$ and $w$, is the radius of the cylinder of conical refraction outside the crystal divided by the beam width. We will present the exact paraxial theory for any $\rho_{0}$, but the interesting regime, for which the phenomena of conical diffraction emerge distinctly, is $\rho_{0} \gg 1$; if $\rho_{0}$ is not large, the fine details are obscured by the beam width.

$\rho$ and $\zeta$ are the dimensionless variables denoting radial position and propagation distance in the field outside the crystal, with $\zeta$ measured from the location $z=l\left(1-1 / n_{2}\right)$ of the virtual image of the incident beam waist in the equivalent isotropic crystal with index $n_{2}$; we call this the focal image plane (figure 4). In physical terms, $\rho$ measures radial position in units of the beam width $w$ and $\zeta$ measures propagation distance in units of the Rayleigh range (Fresnel length) $k_{0} w^{2}$.

For any state of polarization of the incident beam, the emergent field depends on the following two integrals, each depending on two variables and involving Bessel functions $J_{0}$ and $J_{1}$ :

$$
\left.\begin{array}{l}
C_{0}\left(u, u_{0}\right)=\int_{0}^{\infty} \mathrm{d} q q \exp \left\{-\frac{1}{2} q^{2}\right\} \cos \left(u_{0} q\right) J_{0}(u q), \\
C_{1}\left(u, u_{0}\right)=\int_{0}^{\infty} \mathrm{d} q q \exp \left\{-\frac{1}{2} q^{2}\right\} \sin \left(u_{0} q\right) J_{1}(u q) .
\end{array}\right\}
$$

(These integrals are simple transformations of $B_{0}$ and $B_{1}$ in Berry (2004).) 
We will need the formula for the light intensity not for arbitrary incident polarization (e.g. Berry 2004), but only for circularly polarized light:

$$
I\left(\rho, \zeta ; \rho_{0}\right)=\frac{1}{1+\zeta^{2}}\left[\left|C_{0}\left(\frac{\rho}{\sqrt{1+\mathrm{i} \zeta}}, \frac{\rho_{0}}{\sqrt{1+\mathrm{i} \zeta}}\right)\right|^{2}+\left|C_{1}\left(\frac{\rho}{\sqrt{1+\mathrm{i} \zeta}}, \frac{\rho_{0}}{\sqrt{1+\mathrm{i} \zeta}}\right)\right|^{2}\right]
$$

(the formula is also valid for unpolarized light). This is the exact paraxial theory in its simplest form. Although it gives the intensity beyond the crystal, it holds also for the field continued backwards to $\zeta$ values corresponding to points inside the crystal or even in front of it, because such virtual image planes can (and in our experiments will) be made real by the lens beyond the crystal.

For the case $\rho_{0} \gg 1$ in which we are interested, the formulae simplify. Close to the rings, that is when $\left|\rho-\rho_{0}\right| \ll \rho_{0}$ and, provided $\zeta \ll \rho_{0}, C_{0}$ and $C_{1}$ can be approximated as a single function of one variable, namely

$$
C_{0}\left(u, u_{0}\right) \approx C_{1}\left(u, u_{0}\right) \approx \frac{1}{\sqrt{u_{0}}} f\left(u-u_{0}\right),
$$

where, with $K_{\nu}$ and $I_{\nu}$ denoting Bessel functions,

$$
\begin{gathered}
f(s)=\frac{1}{\sqrt{2 \pi}} \int_{0}^{\infty} \mathrm{d} q \sqrt{q} \exp \left(-\frac{1}{2} q^{2}\right) \cos \left(q s-\frac{1}{4} \pi\right) \\
=\frac{1}{4 \sqrt{2 \pi}}|s|^{3 / 2} \exp \left(-\frac{1}{4} s^{2}\right)\left[K_{3 / 4}\left(\frac{1}{4} s^{2}\right)+\operatorname{sgn}(s) K_{1 / 4}\left(\frac{1}{4} s^{2}\right)\right. \\
\left.+\pi \sqrt{2} \Theta(-s)\left(I_{3 / 4}\left(\frac{1}{4} s^{2}\right)-I_{1 / 4}\left(\frac{1}{4} s^{2}\right)\right)\right] .
\end{gathered}
$$

Thus, the intensity of the rings is

$$
I_{\text {rings }}=\frac{2}{\rho_{0}\left(1+\zeta^{2}\right)^{3 / 4}}\left|f\left(\frac{\rho-\rho_{0}}{\sqrt{1+i \zeta}}\right)\right|^{2} .
$$

Figure $5 a-c$ shows how well this approximates the exact paraxial intensity (3.6).

The rings are sharpest in the focal image plane $\zeta=0$ (figure $5 a$ ). Numerical study of (3.9) for $\zeta=0$ shows that the Poggendorff dark ring, where $f=0$, is at $s=-0.765$. The bright rings are the maxima of $f^{2}$, the outer, at $s=+0.551$, having greater intensity, by a factor of 4.735 , than the inner, at $s=-1.764$.

Away from the focal image plane, that is where $s=\left(\rho-\rho_{0}\right) / \sqrt{ }(1+i \zeta)$, further simplification is possible. For $1 \ll \zeta \ll \rho_{0}$, the most elementary Bessel asymptotics gives the geometrical-optics intensity, in which the outer and inner rings originate in the two sheets near the conical intersection of the wave surface:

$$
I_{\text {geom }}\left(\rho, \zeta ; \rho_{0}\right) \approx \frac{\left|\rho-\rho_{0}\right|}{2 \rho_{0} \zeta^{2}} \exp \left(-\frac{\left(\rho-\rho_{0}\right)^{2}}{\zeta^{2}}\right) .
$$

The bright rings, at $\rho=\rho_{0} \pm \zeta / \sqrt{ } 2$, are symmetrical, and centred on the Poggendorff dark ring at $\rho=\rho_{0}$.

More sophisticated asymptotics (explained by Berry (2004)) introduces interference: between the inner ring and a contribution that can be interpreted 

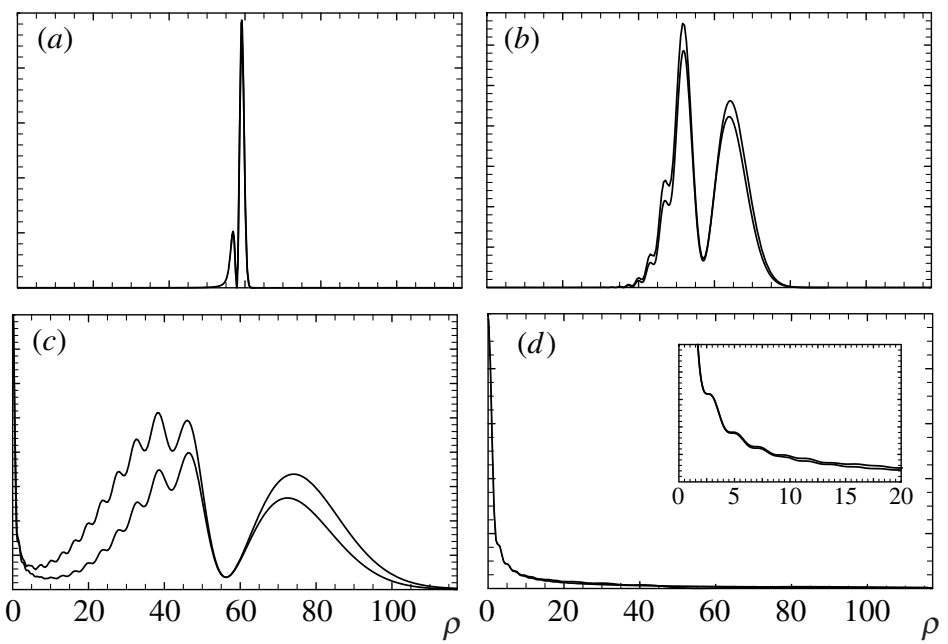

Figure 5. Theoretically calculated intensity profiles for $\rho_{0}=60$, for $(a) \zeta=0 ;(b) \zeta=8 ;(c) \zeta=22$; $(d) \zeta=40$ (the inset in $(d)$ is a magnification of the central spot). Thick curves: intensities computed exactly from (3.6), in terms of the integrals (3.5). Thin curves in $(a)$ and $(b)$ : asymptotic approximation $I_{\text {rings }}(3.9)$. Thin curves in $(d)$ : asymptotic approximation $I_{\text {spot }}(3.13)$. Thin curve in $(c)$ : sum of the asymptotic approximations $I_{\text {rings }}+I_{\text {spot }}$.

as a wave scattered from the conical intersection itself, leading to

$$
\begin{aligned}
I_{\text {rings }}\left(\rho, \zeta ; \rho_{0}\right) \approx & I_{\text {geom }}\left(\rho, \zeta ; \rho_{0}\right) \\
& +\frac{\Theta\left(\rho_{0}-\rho\right)}{4 \rho_{0}\left|\rho_{0}-\rho\right|^{3}}\left[1-\frac{2^{3 / 2}\left|\rho_{0}-\rho\right|^{2}}{\zeta} \exp \left(-\frac{\left(\rho-\rho_{0}\right)^{2}}{2 \zeta^{2}}\right) \cos \left(\frac{\left(\rho-\rho_{0}\right)^{2}}{2 \zeta}\right)\right] .
\end{aligned}
$$

The oscillations are clearly visible in figure $5 b, c$. The correction is valid only when $\rho<\rho_{0}-\sqrt{ } \zeta$, so its divergence at $\rho=\rho_{0}$ is never encountered. The width of the fringes near $\rho$ is of the order

$$
\Delta \rho_{\text {fringe }}=\frac{2 \pi \zeta}{\rho_{0}-\rho} ;
$$

therefore, the fringes get finer as $\rho$ decreases, and there are about $\zeta /(2 \pi)$ of them in the $1 / \mathrm{e}^{2}$ width $\zeta \sqrt{ } 2$ of the inner ring.

As $\zeta$ increases, the outer ring fades away, and the inner ring collapses onto the central spot associated with external conical refraction. In this regime $\left(\zeta>\rho_{0}\right)$, the asymptotics (3.7)-(3.12) is no longer valid, and it is necessary to return to (3.6). Different asymptotics leads to the spot intensity

$$
I_{\text {spot }}\left(\rho, \zeta ; \rho_{0}\right) \approx \frac{\pi \rho_{0}^{2}}{2 \zeta^{3}} \exp \left(-\frac{\rho_{0}^{2}}{\zeta^{2}}\right)\left[J_{0}\left(\frac{\rho \rho_{0}}{\zeta}\right)^{2}+J_{1}\left(\frac{\rho \rho_{0}}{\zeta}\right)^{2}\right] .
$$

This predicts a maximum spot intensity near $\zeta=\rho_{0} \sqrt{ }(2 / 3)$. The function $J_{0}^{2}(x)+$ $J_{1}^{2}(x)$ possesses shoulders - flat inflection points - at the zeros of $J_{1}(x)$, which have the spacing $\Delta \rho \approx \pi \zeta / \rho_{0}$; these are the weak fringes surrounding the central spot, visible in figure $5 d$. 

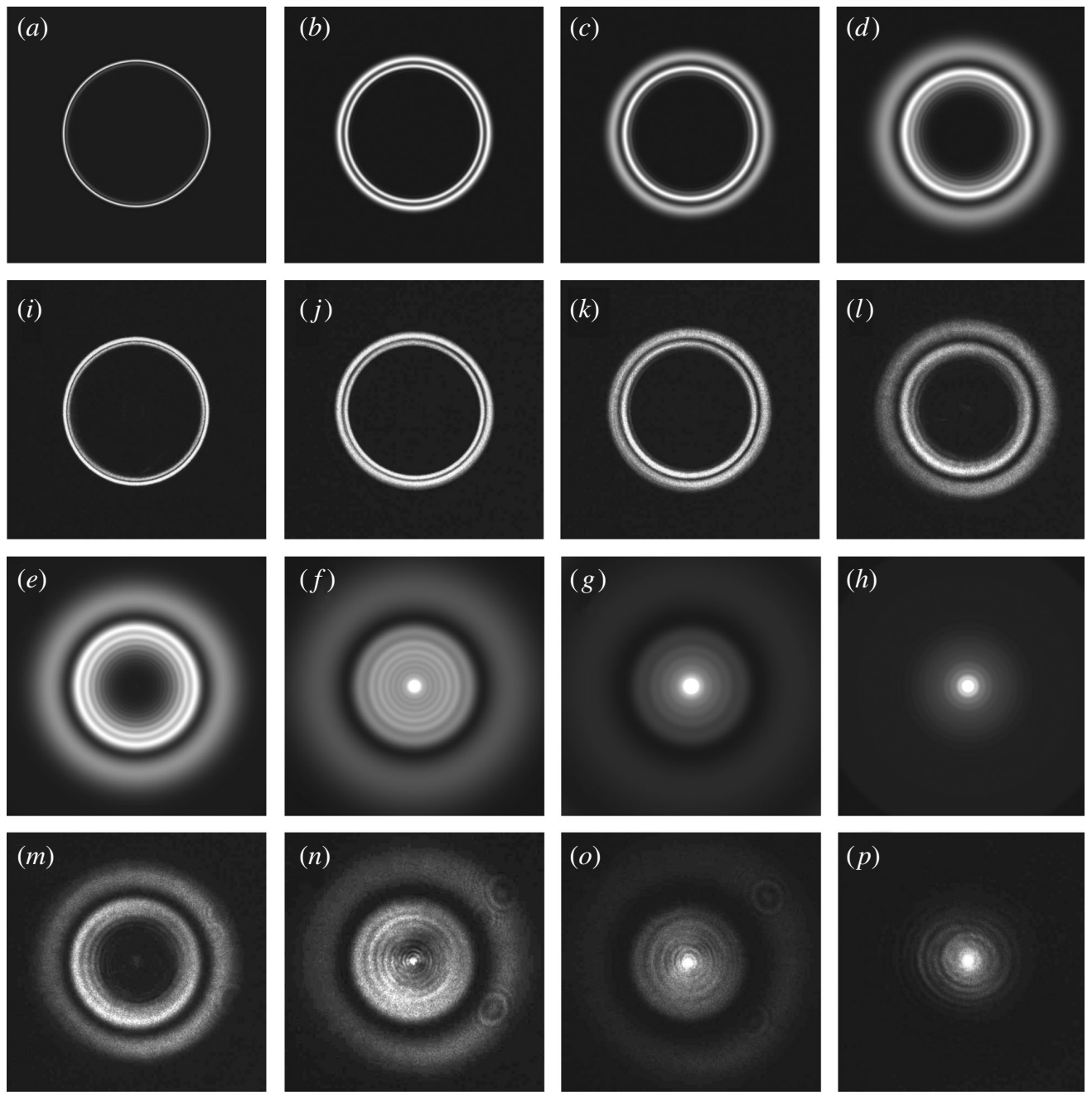

Figure 6. $(a-h)$ Theoretical density plots of conical diffraction intensities at different propagation distances, computed from (3.6), for $\rho_{0}=60$ and $(a) \zeta=0,(b) \zeta=3,(c) \zeta=6,(d) \zeta=12,(e) \zeta=18$, $(f) \zeta=30,(g) \zeta=42,(h) \zeta=98$. $(i-p)$ Experimental conical diffraction patterns corresponding to $(a-h)$, observed at distances $z=k_{0} w^{2} \zeta=0.501 \zeta \mathrm{mm}$ from the focal image plane (the blemishes on the right-hand side of $(n)$ and $(o)$ result from lens imperfections).

\section{Comparison of theory with experiment}

The theoretical conical diffraction patterns depend on the dimensionless quantities defined in (3.4). Most important is the parameter $\rho_{0}$, involving the beam width $w$ of the laser beam focused by the lens $\mathrm{L}_{1}$. We determined $w$ by removing the crystal and the projection lens $\mathrm{L}_{2}$, measuring the width $W$ of the spot on the screen (distance $D$ ), and using the beam-spreading formula $W=\sqrt{w^{2}+\left(D / k_{0} w\right)^{2}} \approx D / k_{0} w$, i.e. $w=D / k_{0} W$. This gave $w=7.1 \pm 0.6 \mu \mathrm{m}$. We were not able to calculate $A$ directly from theory using (3.2), because to our knowledge the three principal refractive indices of our $\mathrm{KGd}\left(\mathrm{WO}_{4}\right)_{2}$ crystal have 

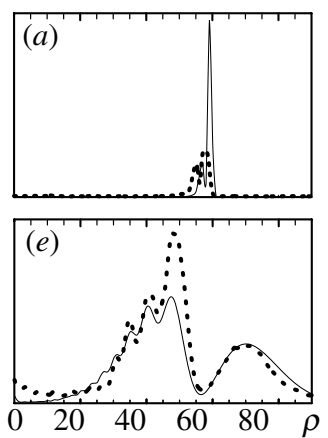
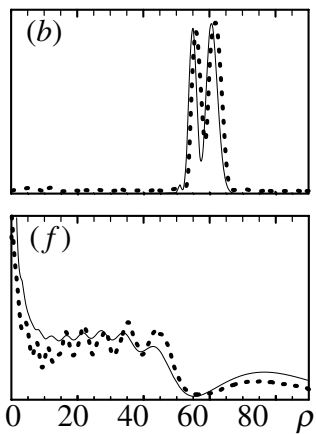
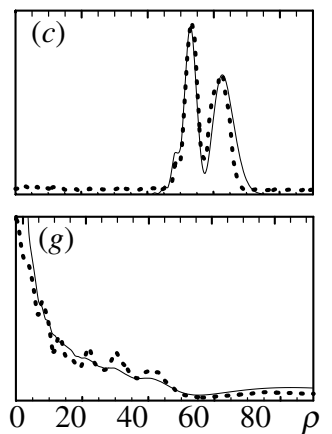
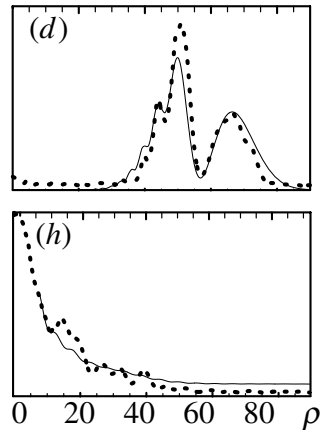

Figure 7. Graphs of radial intensity corresponding to figure 6 for $(a) \zeta=0,(b) \zeta=3,(c) \zeta=6$, (d) $\zeta=12,(e) \zeta=18,(f) \zeta=30,(g) \zeta=42,(h) \zeta=98$. Full curves, theory; dotted curves, experiment. The experimental graphs were obtained by importing the digital photographs into Mathematica as JPEG files, and smoothing over an angular sector of $10^{\circ}$ (using the command ListConvolve). Vertical scales were chosen for best fit.

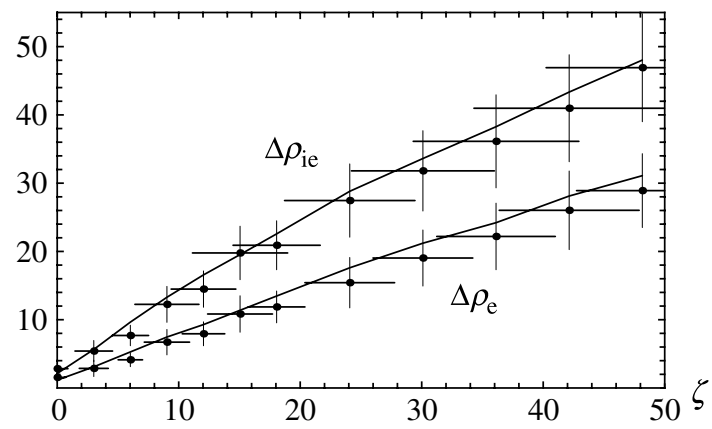

Figure 8. Poggendorff ring dimensions: comparison between theory (full curves) and observations (filled circles). We show distances $\Delta \rho_{\mathrm{e}}$ between external (outer) bright ring maximum and Poggendorff dark ring (of zero intensity), and distances $\Delta \rho_{\text {ie }}$ between the inner and outer bright ring maxima. The errors in $\Delta \rho$ arise from differences between the four azimuth-averaged $10^{\circ}$ sectors of the digital images, and the errors in $\zeta$ arise from the error in $w$.

not been measured accurately enough. Instead, we found the ring radius $A l$ by measuring the radius on the screen of the dark Poggendorff ring (figure $3 a$ ), with the arrangement of figure 1 , as magnified by the lens $\mathrm{L}_{2}$. The result is

$$
\rho_{0}=60 \pm 10 \text {. }
$$

The dimensionless propagation distance $\zeta$ depends sensitively on physical propagation distance $z$. From $(3.4), \Delta \zeta=k_{0} w^{2} \Delta z=1.998 \times \Delta z(\mathrm{~mm})$, so that a change $\Delta \zeta=1$, over which the theoretical pattern changes significantly when $\zeta$ is small, requires an adjustment $\Delta z=0.5 \mathrm{~mm}$.

Figure 6 shows computed and observed ring patterns for different propagation distances. It is clear that the observations display all the features predicted by theory. The only discrepancy is at $\zeta=0$, where theory indicates slightly more sharply focused rings than we were able to obtain. For a more detailed comparison, figure 7 shows the corresponding graphs of intensity as a function of dimensionless radius $\rho$. Overall, the agreement is excellent, but these pictures reveal discrepancies 


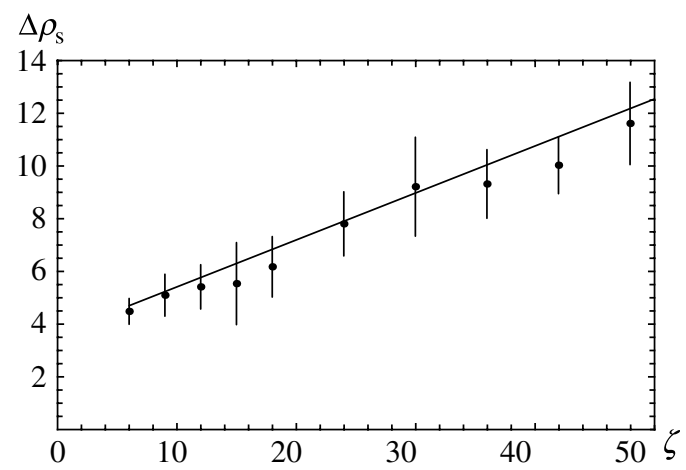

Figure 9. Width of the first secondary diffraction ring, i.e. the distance $\Delta \rho_{\mathrm{s}}$ between the two inner ring maxima closest to the dark ring: comparison between theory (full curve) and experiment (data points).

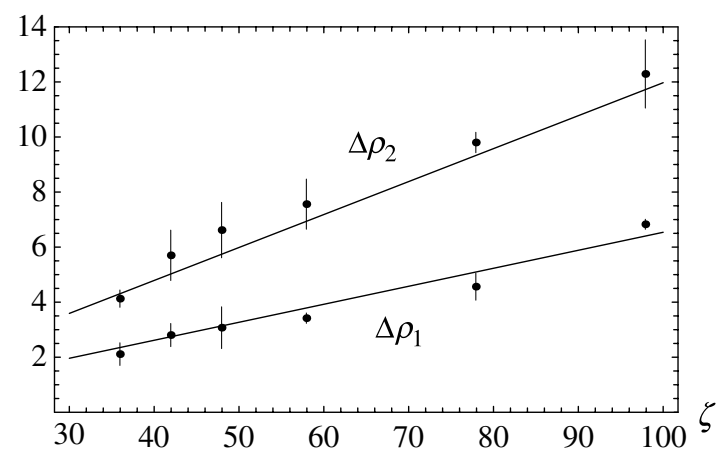

Figure 10. Radii $\Delta \rho_{1}$ (lower) and $\Delta \rho_{2}$ (upper) of the first two diffraction rings surrounding the central spot; full curves: theoretical predictions from shoulders of $J_{0}^{2}(x)+J_{1}^{2}(x)$ in (3.13); data points: measured ring widths.

in the far field $\zeta \gg 1$ : the rings surrounding the central spot have low-contrast maxima and minima rather than the theoretically predicted shoulders.

When $\zeta$ is not too large, the most prominent feature is the separation into two Poggendorff bright rings. Figure 8 is a comparison of the predicted and observed widths of these rings. Again, the agreement is good. In these fits, we employed the exact paraxial diffraction theory (3.6) because the ring separation given by geometrical optics (3.10) is not accurate enough.

Diffraction generates the secondary rings inside the inner Poggendorff bright ring. Figure 9 compares theory and experiment for the separation between the first secondary ring maximum and the main maximum of the inner ring. Again, theory gives a good account of the observations. As is clear from figure $7 d, e$, the secondary rings get narrower as their radius decreases, as predicted by (3.12). The number of secondary rings increases with $\zeta$, in accordance with the prediction after equation (3.12).

Finally, we consider the far field $\zeta \gg 1$. According to (3.13), diffraction rings should lie at radii given by zeros of the Bessel function $J_{1}(x)$, and figure 10 , showing the first two rings, confirms this prediction. 


\section{Concluding remarks}

The experiment reported here confirms the accuracy of the paraxial approximation (Belskii \& Khapalyuk 1978; Berry 2004) as the basis for the simplest quantitative theory incorporating all details of wave and geometrical effects associated with conical diffraction. Paraxiality has two main advantages. First, it enables the pattern beyond the crystal to be described in terms of the single parameter $\rho_{0}$, allowing a fully quantitative comparison with theory without needing to know the three refractive indices. Second, it provides a framework within which both internal and external conical refraction can be comprehended; these are commonly regarded as distinct, but in fact one transforms into the other as the propagation distance increases, a point first made by Raman (1942).

The phenomena of conical diffraction-Poggendorff rings, secondary rings, the central spot and its associated rings - are asymptotically emergent, in the sense that they become more clearly separated as $\rho_{0}$ increases. The value $\rho_{0}=60$ in our experiment was large enough to display this emergence. By replacing the lens $\mathrm{L}_{1}$ (figure 1) with one of longer focal length, it is possible to increase the beam width $w$ and so make $\rho_{0}$ smaller. We made some such observations, and confirmed that the rings get broader and the finer details are less distinct.

Our study has been concerned with the simplest situation, where the biaxial crystal is non-chiral and transparent. There have been some theoretical (Voigt 1905b; Schell \& Bloembergen 1978b; Belsky \& Stepanov 2002) and experimental (Schell \& Bloembergen 1978b) investigations taking account of chirality (optical activity), but recent theoretical study (Berry \& Jeffrey in press) reveals a qualitatively new phenomenon introduced by chirality: beyond the crystal, a geometrical caustic in the form of a cusped cone. The influence of anisotropic absorption (dichroism) remains unexplored; however, the dramatic modifications that dichroism introduces to the wave surface and on the polarization of plane waves in the crystal (Berry \& Dennis 2003) give reason to anticipate some striking associated conical diffraction effects.

M.V.B.'s and M.R.J.'s researches are supported by the Royal Society. We thank Oliver Müller and Thorben Windeler for a helpful correspondence.

\section{Appendix A. Accuracy of paraxial approximation}

Paraxially, the centre of the rings corresponding to internal conical refraction coincides with the spot corresponding to external conical refraction. In the exact geometrical theory, these points, corresponding to directions in space, are distinct, and the smallness of their separation is a measure of paraxiality.

In comparing the exact and approximate angles, it is convenient to define

$$
\alpha=\frac{1}{n_{1}^{2}}-\frac{1}{n_{2}^{2}}, \quad \beta=\frac{1}{n_{2}^{2}}-\frac{1}{n_{3}^{2}} .
$$

The paraxial approximation is $\alpha \ll 1, \beta \ll 1$. We will refer to figure 11, showing the $k_{x}, k_{z}$ plane, with directions of lines emanating from the origin representing wavevector directions, and angles measured from the $k_{z}$ direction. 


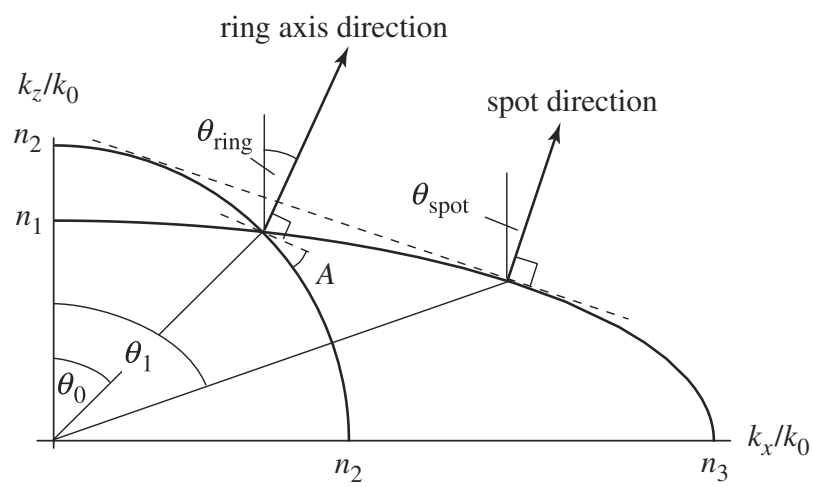

Figure 11. Geometry and notation for ray and wave directions.

The angle of the optic axis (conical intersection of the two wave surfaces) is given, exactly, by Landau et al. (1984)

$$
\tan \theta_{0}=\sqrt{\frac{\alpha}{\beta}} .
$$

The semiangle $A_{\text {exact }}$ of the wave cone, equal to the semiangle of the cone of internal conical refraction, is given by Born \& Wolf (1959)

$$
\tan 2 A_{\text {exact }}=n_{2}^{2} \sqrt{\alpha \beta} .
$$

Paraxially, this is small, being proportional to the geometric mean of the refractive-index differences (cf. equation (3.2)). The direction of the axis of the wave cone, corresponding to the centre of the ring system in the experiments, is

$$
\theta_{\text {ring }}=\theta_{0}-A_{\text {exact }} \text {. }
$$

There are two wave directions corresponding to the contacts of the wave surfaces with their common tangent; the larger of these has its angle given, exactly, by

$$
\tan \theta_{1}=\frac{n_{3}}{n_{1}} \sqrt{\frac{\alpha}{\beta}} .
$$

The normal to this tangent is the direction of the ray (biradial) corresponding to external conical refraction (Landau et al. 1984), which appears as a spot in the experiments:

$$
\tan \theta_{\text {spot }}=\frac{n_{1}}{n_{3}} \sqrt{\frac{\alpha}{\beta}} .
$$

In general, $\theta_{\text {ring }}$ and $\theta_{\text {spot }}$ are different. A paraxial perturbation calculation gives the difference as

$$
\begin{aligned}
\theta_{\text {ring }}-\theta_{\text {spot }}= & \frac{\sqrt{\alpha \beta}}{8} n_{2}^{4}\left[(\beta-\alpha)+\frac{(\alpha+\beta)^{2} n_{2}^{2}}{8}\right]+\cdots \\
= & \frac{\sqrt{\left(n_{2}-n_{1}\right)\left(n_{3}-n_{2}\right)}}{2 n_{2}^{2}}\left[n_{1}+n_{3}-2 n_{2}\right. \\
& \left.+\frac{1}{4 n_{2}}\left(14\left(n_{2}-n_{1}\right)\left(n_{3}-n_{2}\right)-5\left(\left(n_{2}-n_{1}\right)^{2}+\left(n_{3}-n_{2}\right)^{2}\right)\right)\right]+\cdots
\end{aligned}
$$


The leading term is proportional to the square of the refractive-index differences. However, the factors $\beta-\alpha$ and $n_{1}+n_{3}-2 n_{2}$ indicate that if the two index differences are comparable, then this term can vanish; therefore, we have included the next (cubic) correction. Some algebra gives the condition for the directions to coincide exactly, that is, to all post-paraxial orders:

$$
\theta_{\text {ring }}=\theta_{\text {spot }} \Rightarrow n_{1}^{2}+n_{3}^{2}=2 n_{2}^{2} \text {. }
$$

To estimate the paraxial errors, we choose two examples. First is the monoclinic double tungstate crystal $\mathrm{KYb}\left(\mathrm{WO}_{4}\right)_{2}$, similar to that in our experiment but with $\mathrm{Yb}$ instead of Gd, for which the indices have been measured (Pujol et al. 2002); we find

$$
\left.\begin{array}{c}
n_{1}=2.02139, \quad n_{2}=2.06494, \quad n_{3}=2.11175, \\
\alpha=0.010214, \quad \beta=0.010282, \\
A_{\text {exact }}=0.999 A=0.02183=1.251^{\circ}, \\
\theta_{\text {ring }}-\theta_{\text {spot }}=0.00103 A=4.63 \text { arcsec. }
\end{array}\right\}
$$

This indicates that the central spot deviates from the centre of the ring by $0.1 \%$, so the paraxial approximation is excellent. Note that $\alpha$ and $\beta$ are comparable in magnitude as well as small, so this crystal falls into the class where the deviation is determined by the cubic terms in (A 7).

A more discriminating test is naphthalene, because this gives the largest rings so far reported (Raman et al. 1941). The relevant quantities are

$$
\left.\begin{array}{c}
n_{1}=1.525, \quad n_{2}=1.722, \quad n_{3}=1.945, \\
\alpha=0.09276, \quad \beta=0.07290, \\
A_{\text {exact }}=0.983 A=0.1196=6.852^{\circ}, \\
\theta_{\text {ring }}-\theta_{\text {spot }}=0.01465 A=6.02 \text { arcmin. }
\end{array}\right\}
$$

Even here, the deviation is only about $1.5 \%$.

These estimates justify the paraxial approximation for all reported experiments on conical diffraction.

\section{References}

Belskii, A. M. \& Khapalyuk, A. P. 1978 Internal conical refraction of bounded light beams in biaxial crystals. Opt. Spectrosc. (USSR) 44, 436-439.

Belsky, A. M. \& Stepanov, M. A. 1999 Internal conical refraction of coherent light beams. Opt. Commun. 167, 1-5. (doi:10.1016/S0030-4018(99)00251-5)

Belsky, A. M. \& Stepanov, M. A. 2002 Internal conical refraction of light beams in biaxial gyrotropic crystals. Opt. Commun. 204, 1-6. (doi:10.1016/S0030-4018(02)01191-4)

Berry, M. V. 2000 Making waves in physics: three wave singularities from the miraculous 1830s. Nature 403, 21. (doi:10.1038/47364)

Berry, M. V. 2004 Conical diffraction asymptotics: fine structure of Poggendorff rings and axial spike. J. Opt. A 6, 289-300. (doi:10.1088/1464-4258/6/4/001) 
Berry, M. V. \& Dennis, M. R. 2003 The optical singularities of birefringent dichroic chiral crystals. Proc. R. Soc. A 459, 1261-1292. (doi:10.1098/rspa.2003.1155)

Berry, M. V. \& Jeffrey, M. R. In press. Chiral conical diffraction.

Berry, M. V., Jeffrey, M. R. \& Mansuripur, M. 2005 Orbital and spin angular momentum in conical diffraction. J. Opt. A 7, 685-690. (doi:10.1088/1464-4258/7/11/011)

Born, M. \& Wolf, E. 1959 Principles of optics. London: Pergamon Press.

Dreger, M. A. 1999 Optical beam propagation in biaxial crystals. J. Opt. A: Pure Appl. Opt. 1, 601-616. (doi:10.1088/1464-4258/1/5/305)

Fève, J. P., Boulanger, B. \& Marnier, G. 1994 Experimental study of internal and external conical refraction in KTP. Opt. Commun. 105, 243-252. (doi:10.1016/0030-4018(94)90722-6)

Hamilton, W. R. 1837 Third supplement to an essay on the theory of systems of rays. Trans. $R$. Irish Acad. 17, 1-144.

Landau, L. D., Lifshitz, E. M. \& Pitaevskii, L. P. 1984 Electrodynamics of continuous media. Oxford: Pergamon Press.

Lloyd, H. 1833 On the phenomena presented by light in its passage along the axes of biaxial crystals. Phil. Mag. 1, 112-120 and 207-210.

Lloyd, H. 1837 On the phenomena presented by light in its passage along the axes of biaxial crystals. Trans. R. Irish Acad. 17, 145-158.

Mikhailichenko, Y. P. 2004 Large scale experiments on conical refraction. Available at http:// www.demophys.tsu.ru/Original/Hamilton/Hamilton.html.

O'Hara, J. G. 1982 The prediction and discovery of conical refraction by William Rowan Hamilton and Humphrey Lloyd (1832-1833). Proc. R. Irish Acad. 82A, 231-257.

Perkalsris, B. S. \& Mikhailichenko, Y. P. 1979 Demonstration of conical refraction. Izv. Vyss. Uch. Zav. Fiz. 8, 103-105.

Poggendorff, J. C. 1839 Ueber die konische refraction. Pogg. Ann. 48, 461-462.

Pujol, M. C. et al. 2002 Growth, optical characterization, and laser operation of a stoichiometric crystal KYb $\left(\mathrm{WO}_{4}\right)_{2}$. Phys. Rev. B 65, 165121. (doi:10.1103/PhysRevB.65.165121)

Raman, C. V. 1942 The phenomena of conical refraction. Curr. Sci. 11, 44-46.

Raman, C. V., Rajagopalan, V. S. \& Nedungadi, T. M. K. 1941 Conical refraction in naphthalene crystals. Proc. Indian Acad. Sci. A14, 221-227.

Schell, A. J. \& Bloembergen, N. 1978a Laser studies of internal conical diffraction. I. Quantitative comparison of experimental and theoretical conical intensity distribution in aragonite. J. Opt. Soc. Am. 68, 1093-1098.

Schell, A. J. \& Bloembergen, N. $1978 b$ Laser studies of internal conical diffraction. II. Intensity patterns in an optically active crystal, $\boldsymbol{\alpha}$-iodic acid. J. Opt. Soc. Am. 68, 1098-1106.

Voigt, W. 1905 a Bemerkung zur theorie der konischen refraktion. Phys. Z. 6, 672-673.

Voigt, W. $1905 b$ Theoretisches unt experimentelles zur Aufklärung des optisches Verhaltens aktiver Kristalle. Ann. Phys. 18, 645-694.

Warnick, K. F. \& Arnold, D. V. 1997 Secondary dark rings of internal conical refraction. Phys. Rev. E 55, 6092-6096. (doi:10.1103/PhysRevE.55.6092) 